\title{
Temporal feature estimation during walking using miniature accelerometers: an analysis of gait improvement after hip arthroplasty
}

\author{
K. Aminian ${ }^{1} \quad$ K. Rezakhanlou ${ }^{1}$ \\ P.-F. Leyvraz ${ }^{2}$ \\ E. De Andres ${ }^{2}$ \\ P. Robert ${ }^{1}$ \\ C. Fritsch ${ }^{2}$ \\ ${ }^{1}$ Swiss Federal Institute of Technology Lausanne (EPFL), Metrology Laboratory, Lausanne, \\ Switzerland \\ ${ }^{2}$ Hôpital Orthopédique de la Suisse Romande, Lausanne, Switzerland
}

\begin{abstract}
A new method for the detection of gait cycle phases using only two miniature accelerometers together with a light, portable digital recorder is proposed. Each subject is asked to walk on a walkway at his/her own preferred speed. Gait analysis was performed using an original method of computing the values of temporal parameters from accelerometer signals. First, to validate the accelerometric method, measurements are taken on a group of healthy subjects. No significant differences are observed between the results thus obtained and those from pressure sensors attached under the foot. Then, measurements using only accelerometers are performed on a group of 12 patients with unilateral hip osteo-arthritis. The gait analysis is carried out just before hip arthroplasty and again, three, six and nine months afterwards. A mean decrease of $88 \%$ of asymmetry of stance time and especially a mean decrease of $250 \%$ of asymmetry of double support time are observed, nine months after the operation. These results confirm the validity of the proposed method for healthy subjects and its efficiency for functional evaluation of gait improvement after arthroplasty.
\end{abstract}

Keywords-Accelerometry, Gait analysis, Orthopaedic, Portable device

Med. Biol. Eng. Comput., 1999, 37, 686-691

\section{Introduction}

NOWADAYS, APPRECIATION of the incidence of physical handicaps and post-operative results after arthroplasties is often based on subjective estimation of the clinical evaluation. This does not reflect the real functional capacity of the patient in his/her usual activity. Considering this, several authors have established questionnaires from which functional scores are developed. Although the questions on such a scale are standardised, the answers are often subjective and the discrepancy between the patient's and the physician's evaluations is significant (LIEBERMAN et al., 1996; ANDERSON, 1972; LIANG et al., 1990).

Because of the lack of objectivity and reproducibility, other scientific studies have developed analysis of the specificity of the different phases of the gait cycle. The time span of each phase of the gait cycle gives the temporal parameters. Evaluation of temporal parameters during walking is a means, not only to assess some aspects of pathological walk among osteo-arthritic patients, but also to quantify their subsequent improvement after hip or knee arthroplasty (WALL et al., 1981; GIANNINI et al., 1994). Temporal parameters are also used to predict force parameters of gait as an alternative to direct ground reaction

Correspondence should be addressed to Dr K. Aminian; email: kamiar.aminian@epfl.ch

First received 26 April 1999 and in final form 16 August 1999

(C) IFMBE: 1999 force measurements for determining daily histories of habitual lower limb loading quantities (BREIT and WHALEN, 1997). In addition to gait analysis, temporal parameters are useful in several clinical fields, such as electrical stimulation (GRAUPE et al., 1987), prediction of future fall (MAKI, 1997) and estimation of metabolic rate (HOYT et al., 1994).

Complete gait analysis, using a video camera (FERRIGNO et $a l ., 1985)$, a walkway with implanted sensors or a force-plate (HIROKAWA and MATSUMARA, 1987; MACELLARI and GIACOMOZZI, 1996), allows these measurements but requires a dedicated laboratory. Ambulatory systems using foot switches or pressure sensors attached to the sole are used for the monitoring of temporal parameters (ROSENROT 1982; ZHU et al., 1991; ABU-FARAJ et al., 1997). These techniques present, in general, satisfactory results in normal walking. However, in pathological gait, many problems, such as shuffling, difficulty of appropriate positioning, connecting attachment, mechanical failure and patient acceptance, render their use limited.

To compensate for the lack of information concerning pathological gait, an analysis based on accelerometry has proved to be an excellent alternative to establish the real individual characteristics of movement in daily life conditions (AMINIAN et al., 1998). In recent years, technical progress has made it possible to realise miniature acceleration sensors with integrated conditioning and a calibrating module. In addition, owing to very low consumption, these accelerometers can be supplied by battery and are a promising tool for long term ambulatory monitoring. The possibility to detect, during 
Table 1 Characteristics of 12 patients

\begin{tabular}{|c|c|c|c|c|c|c|c|c|c|c|c|c|}
\hline Patients & 1 & 2 & 3 & 4 & 5 & 6 & 7 & 8 & 9 & 10 & 11 & 12 \\
\hline Sex (M: male, F: female) & M & M & $\mathrm{M}$ & $\mathrm{F}$ & $\mathrm{F}$ & M & $\mathbf{F}$ & $M$ & $\mathrm{~F}$ & $\mathrm{M}$ & $M$ & $M$ \\
\hline Age, years & 74 & 55 & 62 & 55 & 76 & 76 & 54 & 65 & 61 & 55 & 71 & 70 \\
\hline Weight, $\mathrm{kg}$ & 80 & 91 & 102 & 73 & 70 & 87 & 63 & 86 & 74 & 87 & 80 & 82 \\
\hline Height, $\mathrm{cm}$ & 170 & 177 & 177 & 161 & 156 & 177 & 162 & 180 & 165 & 180 & 168 & 174 \\
\hline BMI, $\mathrm{kg} \mathrm{m}^{-2}$ & 27.7 & 29.0 & 32.6 & 28.2 & 28.8 & 27.8 & 24.0 & 26.5 & 27.2 & 26.9 & 28.3 & 27.1 \\
\hline Hip & left & left & right & right & left & left & left & left & right & right & left & right \\
\hline Pain before surgery, months & 4 & 36 & 18 & 360 & 60 & 18 & 120 & 48 & 12 & 168 & 60 & 360 \\
\hline
\end{tabular}

normal gait, simple parameters such as step and cycle time from trunk or heel acceleration has already been shown (EVANS et al., 1991; AMINIAN et al., 1995).

In this paper, an original method to compute precisely the value of temporal parameters using accelerometry is proposed. It has been successfully applied to assess some aspects of pathological walk among osteo-arthritic patients and to quantify their subsequent improvement after hip arthroplasty.

\section{Method}

\subsection{Measurement}

An accurate measurement of lower-limb acceleration signals, namely thigh acceleration, needs to be recorded for successful determination of temporal parameters. Two piezoresistive accelerometers and a light, portable measuring device Physilog were used to achieve this task. The two uni-axial accelerometers were attached to each leg, slightly above the knee articulation, and measured the tangential component of each thigh acceleration in the sagittal plane. These accelerometers (IC sensors 3021) are accurate, miniature and inexpensive; they have a relatively wide frequency range $(0-350 \mathrm{~Hz})$ and measure up to $\pm 5 \mathrm{~g}$. The signals were digitised (12 bits) by a portable datalogger* and stored on a 1 Mbyte SRAM memory card (AMINIAN et al., 1995). At the end of each recording session, the data in the memory card were transferred to a computer for analysis and evaluation.

First, to validate the accelerometric method, measurements were taken from a group of five healthy subjects. In addition to accelerometers, two FSR pressure sensors $†$ were placed inside each shoe, under the heel and the first metatarsal (big toe). In this way, the moments of heelstrikes and toe-offs were directly detected. Each pair of sensors was connected in series, and the output was simultaneously digitised and recorded by Physilog, with those from accelerometers, at a sampling rate of $60 \mathrm{~Hz}$. An additional, similar test was performed with a patient suffering from hip osteo-arthritis, to compare the accelerometric method with the FSR during a pathological gait.

Next, measurements using only accelerometers were taken from a group of 12 patients with unilateral hip osteo-arthritis . 30 healthy subjects (45-77 years, $51-91 \mathrm{~kg}, 1.5-1.86 \mathrm{~m}$ ) were used as a control group. Each subject walked at his/her own preferred speed. The walkway, $35 \mathrm{~m}$ long, was covered twice for each experiment and was marked at $5 \mathrm{~m}$ intervals. A switch connected to the Physilog recorded the time needed to walk $5 \mathrm{~m}$ and estimated the speed of walking. Hip arthroplasty was carried out on the patients. Table 1 shows the characteristics of each patient. The gait analysis was carried out just before the operation and again, three, six and nine months afterwards.

\subsection{Algorithm}

To compute temporal parameters, such as duration of swing and total or double stances of a gait cycle, it is necessary and sufficient to determine for each leg the precise moments of heelstrike (when the foot first touches the floor) and toe-off (when it last takes off) during that cycle. These events give rise to distinctive features on thigh acceleration signals in the form of rather sharp negative peaks. Although the amplitude of these peaks can, for example, depend on the subject's velocity, weight or painful articulation (in the case of arthritic patients for instance), they are nonetheless always locatable as long as it is known where to look.

Successfully to achieve this task, we should bear in mind the following observations. The swing phase of a gait cycle is characterised by a positive thigh acceleration that reaches its highest values at around mid-swing (see Fig. 1). This derives from the fact that the subject pushes the swinging leg forward, while taking support on the other leg. Prior to the swing phase, in a quick movement backward involving hip and knee articulations, the foot is taking off the floor to avoid scuffling or hitting the floor. As a result, a sharp negative acceleration can be observed that is associated with toe-off. At the end of the swing period, the leg is brought to a halt and put on the floor. This is the heelstrike and is also characterised by a sharp negative acceleration.

Taking advantage of these facts, we have developed an algorithm along the same lines that can be seen on the flow chart of Fig. 2. The algorithm is designed to extract from the right and left thigh acceleration $a_{r}$ and $a_{l}$, recorded during walking by Physilog, four series of points in time. These series contain the precise instances of heelstrikes and toe-offs of the right and left feet. For clarity, let us consider $a_{r}$ as the input. It is obvious that $a_{l}$ will be treated in the same way. We begin by identifying the time corresponding to the global maximum values $p_{s}$ of the smoothed thigh acceleration signal $a_{r f 1}$ (lowpass filtering at $3 \mathrm{~Hz}$ ). The $p_{s}$ samples represent approximately the moment of mid-swing during a gait cycle; however, their exact significance is not of interest to us. They will only be used as a reference to compute the average gait cycle $g c_{m}$ and to select the intervals in which negative peaks reminiscent of heelstrikes and toe-offs are to be found. Therefore we next look for local minima of $a_{r f}$ inside intervals $\left[p_{s}+0.15 g c_{m}, p_{s}+0.3 g c_{m}\right]$ and $\left[p_{s}-0.15 g c_{m}, p_{s}-0.3 g c_{m}\right]$. The result is two different series of samples $p_{h 1}$ (heelstrikes) and $p_{t 1}$ (toe-offs), which is almost what we are looking for. However, we ultimately want to locate these moments on the unfiltered signal $a_{r}$, as, in filtering, a slight shift may have occurred. To do so, we proceed in two separate but similar steps. First, we apply a new low-pass filter at $6 \mathrm{~Hz}$ to $a_{r f 1}$ and we detect the minima of this new function $a_{r f 2}$ in the close vicinity of $p_{h 1}$ and $p_{t 1}$. Two new sample series $p_{h 2}$ and $p_{t 2}$ are

\footnotetext{
*Physilog, BioAGM, CH

†Interlink, LU
} 


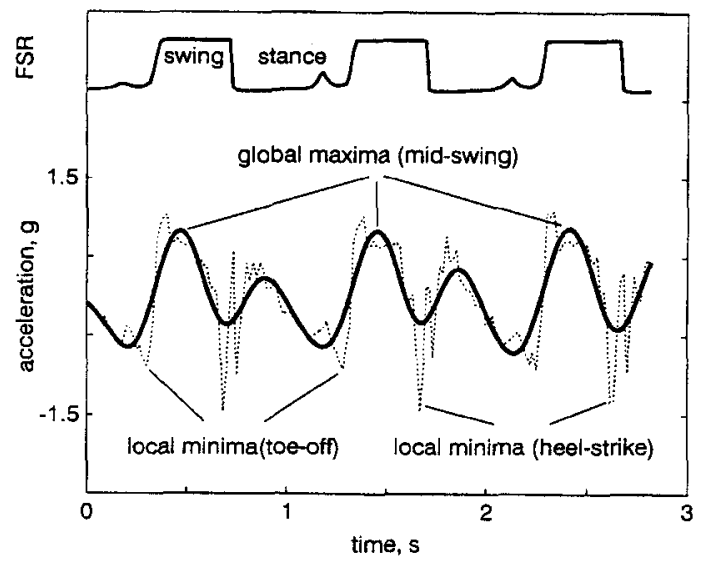

Fig. 1 Raw and filtered thigh acceleration compared with output of FSR sensors during two gait cycles. (. . .) Raw signal; (-) filtered signal

thus obtained. Then, we look at $a_{r}$ in the immediate vicinity of $p_{h 2}$ and $p_{t 2}$ to find the minimum peaks. The result is the final time samples $p_{h r}$ and $p_{t r}$.

This process of gradually focusing on the exact position of heelstrikes and toe-offs on the unfiltered thigh acceleration signal can be carried out, if necessary, in more than one intermediate step. Once we are in possession of $p_{h l}$ (left heelstrike), $p_{t l}$ (left toe-off), $p_{h r}$ (right heelstrike) and $p_{t r}$ (right toe-off), every temporal parameter of a gait cycle can easily be computed as a percentage of gait cycle $g c$ averaged over $N$ consecutive gait cycles. These parameters are

(a) duration of each gait cycle

$$
g c(i)=p_{h r}(i+1)-p_{h r}(i) \quad 1 \leq i \leq N
$$

(b) left stance (time between left heelstrike and left toe-off)

$$
L S(i)=p_{t l}(i)-p_{h l}(i)
$$

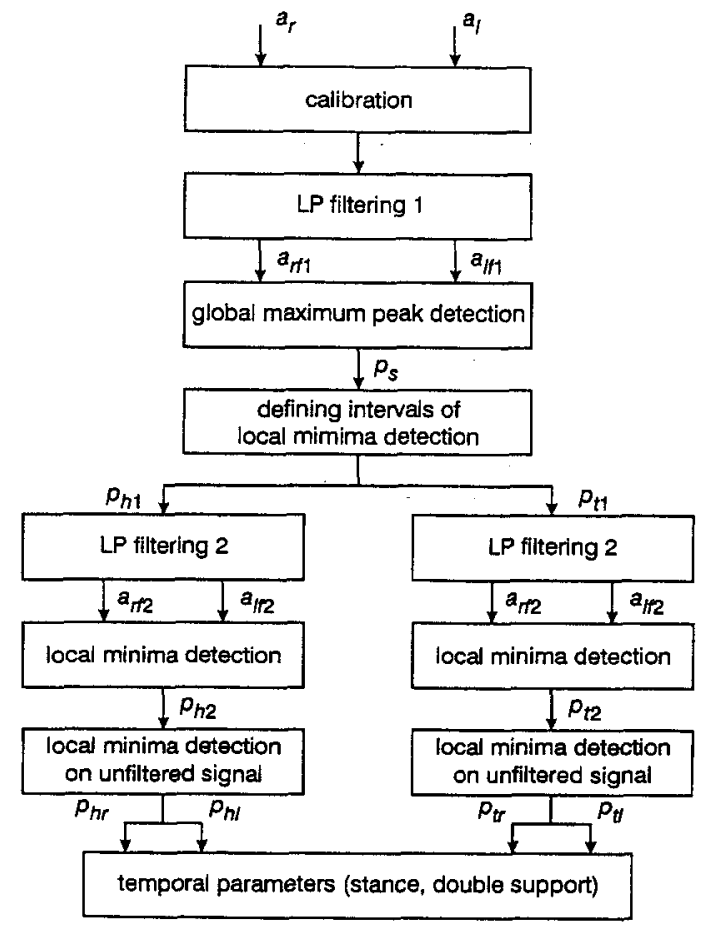

Fig. 2 Flowchart of temporal feature detection (c) right stance (time between right heelstrike and right toe-off)

$$
R S(i)=p_{t r}(i)-p_{h r}(i)
$$

(d) left double support (time between right heelstrike and left toe-off), also known as left double thrust support time (WALL et al.)

$$
L D S(i)=p_{t l}(i)-p_{h r}(i)
$$

(e) right double support (time between left heelstrike and right toe-off), also known as right double thrust support time (WALL et al.)

$$
R D S(i)=p_{\mathrm{tr}}(i)-p_{h l}(i)
$$

To achieve a good level of precision for these parameters, 20 or so gait cycles are needed. This means that the subject on whom we carry out our study is asked to walk at his/her preferred speed for at least $30 \mathrm{~m}$ in a straight line. An increase in this distance might improve the accuracy of the measurement, but, from a clinical point of view, walking $30 \mathrm{~m}$ is reasonable for gait analysis in orthopaedic patients.

\section{Results}

\subsection{Detection algorithm}

Using the algorithm of Fig. 2, left and right heelstrikes (toeoffs) were detected from thigh accelerations, and the values of $L S$ and $R S$ were computed for 16 consecutive gait cycles $(N=16)$. Simultaneously, the period of stance was detected from the FSR signal (Fig. 1), and the corresponding left and right stances over a period of 16 cycles were computed. Fig. 3 compares values obtained from accelerations (ACC) with those of FSR. A good agreement $(R=0.97)$ for both the left and right stance is found. The difference between ACC and FSR for normal subjects is not significant $(N=80, p>0.8)$, and the $95 \%$ confidence interval on the mean is: $-0.02 \mathrm{~s}<$ mean (ACC)-mean(FSR) $<0.02 \mathrm{~s}$. The averaged values of LS and RS, in $\mathrm{s}$, and as a percentage of the gait cycle are presented in Table 2 .

The efficiency of the accelerometric method during pathological gait, tested on one patient, was compared with the pressure sensors. Fig. 4 show two examples of walking, where the FSR signal provides erroneous results, whereas temporal parameters are correctly assessed from accelerations. In Fig. $4 a$, the first and third swing obtained from the FSR signal are approximately the

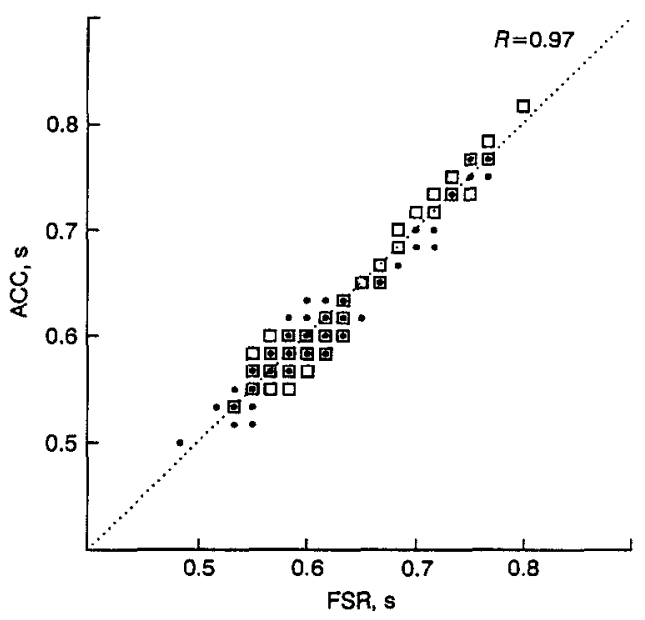

Fig. 3 Right stance (RS) and left stance (LS) duration, in s, obtained from accelerometry (ACC) compared with that obtained from pressure sensors (FSR). (@) $L S$; ( $\square) R S$ 
same and correspond to those obtained from the acceleration signals. However, the pressure sensors detect that the second swing has dropped to half its value, although there is no significant change in the acceleration patterns. This result could be caused by shuffling at the beginning of the swing inducing some pressure on the FSR that does not interfere with the thigh acceleration. In Fig. $4 b$, an artefact is present during the first stance in the FSR signal, owing probably to the fact that the foot was not acting properly on the pressure sensors, involving, during a short time, a strain drop on these sensors. The thigh acceleration was not affected by this artefact.

\subsection{Hip osteo-arthritis patient analysis}

The detection algorithm was then applied to data from 30 normal subjects. The results were averaged over 20 consecutive gait cycles to estimate the normal range of temporal parameters. These normal ranges (mean \pm s.d.), defined as a percentage of the gait cycle, are as follows:

$$
\begin{aligned}
& 59 \% \leq R S, L S \leq 63 \% \\
& 9 \% \leq R D S, L D S \leq 13 \%
\end{aligned}
$$

These values correspond to the established values for the normal population, which are around $60 \%$ for the duration of stance and $10 \%$ for the duration of double support, and remain largely unchanged throughout most adult life (ROSE and GAMBLE, 1994).

Stance and double support time were also detected from the 12 patients before the operation and at 3,6 and 9 months following the operation. Figs. 5 and 6 show these values (averaged over 20 consecutive gait cycles) compared with normal data. By plotting RS against LS, normal data are distributed around the diagonal and centred about $60 \%$, whereas most of the pre-operative data are spread far from the normal data (Fig. $5 a$ ). After the operation, post-operative data move towards the normal data and, at 6 months, nearly reach the normal values. The post-operative improvements are better shown with the variation in RDS and LDS. The pre-operative data are spread generally, far from the diagonal, depending on the degree of pathology (Fig. 6a). In
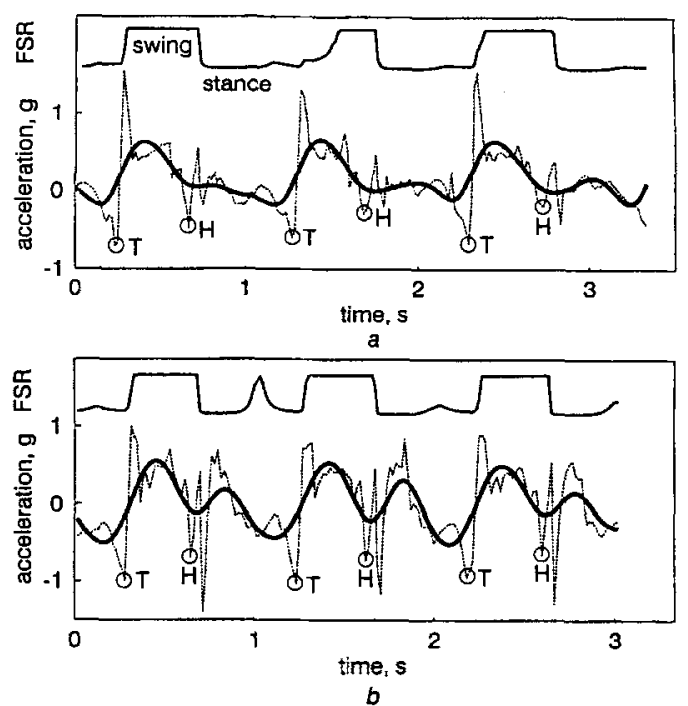

Fig. 4 Two examples where FSR signal has not precisely detected swing and stance phase during pathological gait, whereas toe-offs $(T)$ and heelstrikes $(H)$ are correctly recognised from thigh accelerations. (a) FSR signal seems to measure a pressure during beginning of second swing, probably caused by shuffling. (b) FSR signal measures artefact during first stance. (...) raw signal; (-) filtered signal addition, data from patients with an arthritic left hip are distributed above the diagonal, whereas data from patients with an arthritic right hip are mostly below the diagonal. This is owing to the asymmetry of pathology: RDS (LDS) is shorter than LDS (RDS) for patients with an arthritic right (left) hip. This discrepancy decreases strongly 3 months after the operation (Fig. 6b) and becomes almost normal after 9 months (Fig. $6 d$ ).

A student's $t$-test was performed on the data of the normal population $\left(N_{1}=30\right)$ and of patients $\left(N_{2}=12\right)$ before and after the operation. According to this analysis, pre-operative data (RS, LS, RDS, LDS) are significantly different from the normal data $(p<0.005)$. For all the patients, the speed of walking before and after the operation and the difference between right and left double support |RDS-LDS| are shown in Fig. 7. The speed changes significantly only 6 months after the operation $(p<0.005)$, whereas |RDS-LDS| becomes significantly different 3 months after the operation ( $p<0.05$ at 3 months, and $p<0.005$ at 6 months and 9 months). Furthermore, $\mid$ RS-LS| changes significantly from the pre-operative only after 9 months $(p<0.10)$.

\section{Discussion and conclusions}

Comparisons between the normal population and patients on the one hand and pre-operative and post-operative patients on the other hand are carried out from temporal parameters obtained at a speed freely selected by the subject. This is because, at a freely selected speed, people walk in a dynamically equivalent manner (CHAN and CHILDRESS, 1991). This is shown in Fig. 8, where the difference between left and right double support is presented as a function of the freely selected speed for a normal subject. This difference is less than $2 \%$, which is not significant compared with the resolution of the measurement. In addition, as illustrated in Fig. 7, the change in the freely selected speed is not a powerful indicator of gait ability, as the relative increase in speed after nine months and over 12 patients is only $25 \%$, whereas, for the same period, a decrease of $88 \%$ in the asymmetry of the stance time $\mid$ RS-LS $\mid$ and, especially, a decrease of $250 \%$ in the asymmetry of the double support time
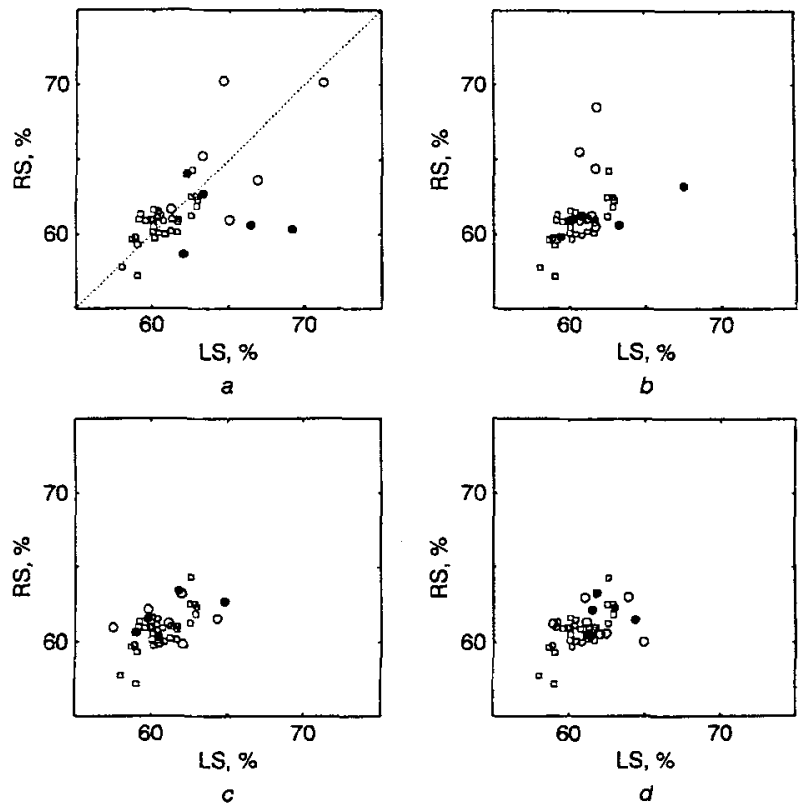

Fig. 5 Right stance (RS) against left stance (LS) as percentage of gait cycle: (a) before operation; (b) 3 months after operation; (c) 6 months after operation; (d) 9 months after operation. (O) Left; (@) right; ( $\square$ ) normal 

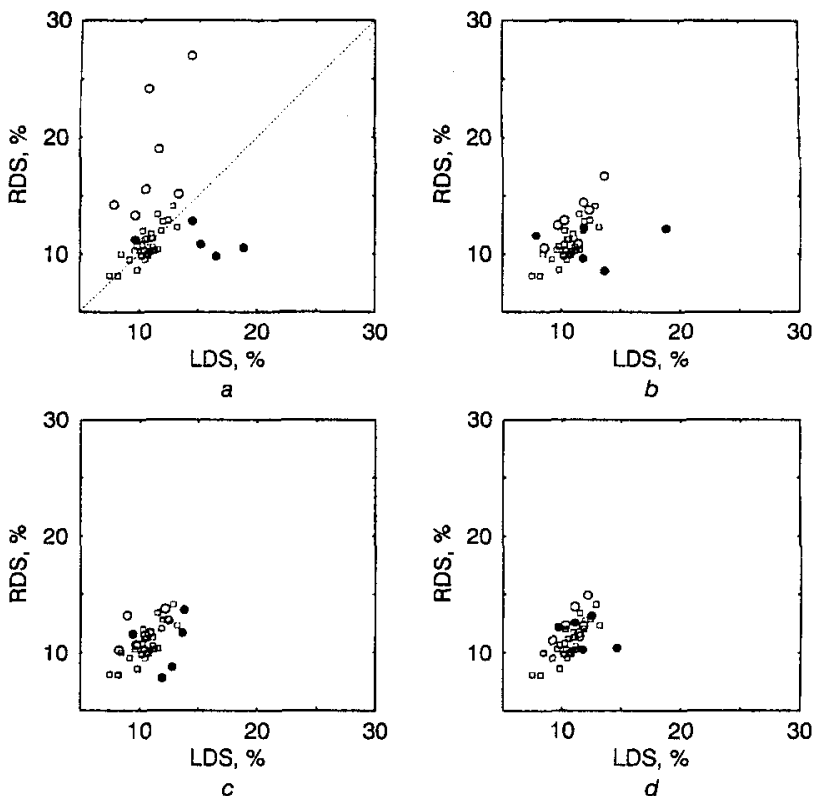

Fig. 6 Right double support (RDS) against left double support (LDS) as percentage of gait cycle: (a) before operation; (b) 3 months after operation; (c) 6 months after operation; (d) 9 months after operation. (O) Left; ( $)$ right; ( $\square)$ normal

|RDS-LDS| were observed. It can be concluded that the double support time is the temporal parameter that allows control of gait symmetry recovery during rehabilitation. Any discrepancy between the durations of left and right double limb support depends on the affected hip. Right osteo-arthritic patients have a left double support longer than the right. The reverse is true for left osteo-arthritic patients. The recovery of symmetry is progressive for every patient. The contrast with the almost equal values for a population of normal subjects is pronounced. This result underlines that the weight transfer phase is the most demanding task during the gait cycle and certainly a painful one for arthritic patients. Furthermore, a tendency towards normality was also observed after arthroplasty.

If we consider patients $3,4,5,7$ and 10 (Fig. 7b), |RDS-LDS| shows no progression after arthroplasty and is already low before arthroplasty (maximum 5\%, patient 3 ). The clinical
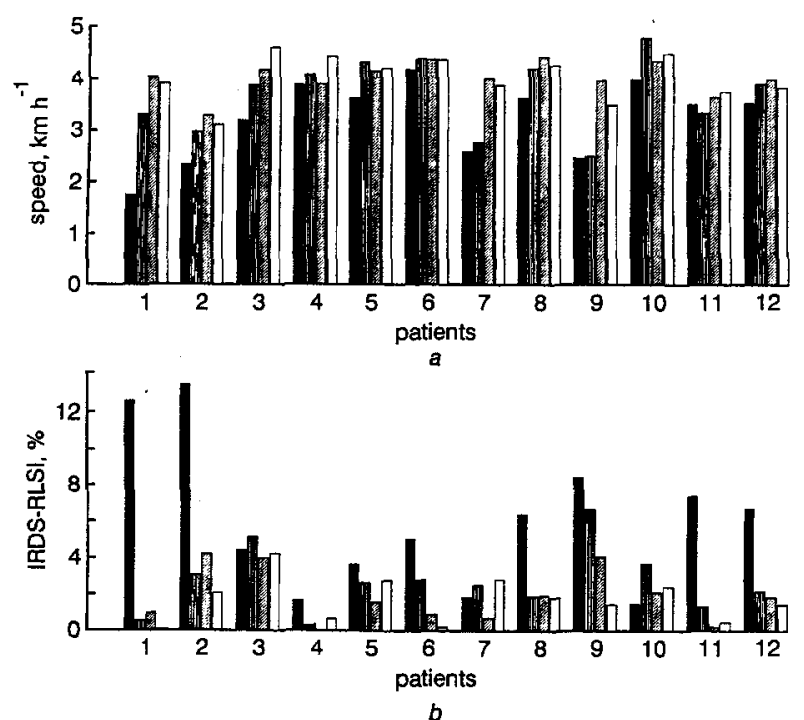

Fig. 7 (a) Speed and (b) $|R D S-L D S|$ variation after operation for all patients. (回) Before operation; (四) 3 months, (《Z) 6 months, ( $\square$ ) 9 months

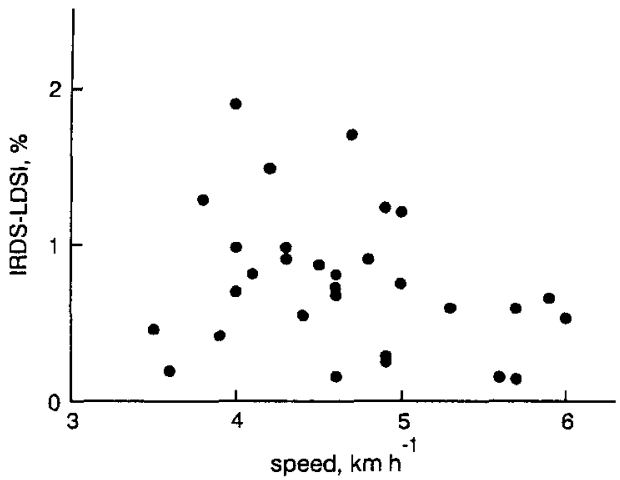

Fig. 8 Variation of $|R D S-L D S|$ as functions of freely selected speed of walking for normal subject

evidence regarding this result is as follows: first, all patients had a discrete (unnoticeable unless looked for) limp, except patient 3 who had a moderate (anybody could notice it) limp before surgery; secondly, these patients are younger (mean age 60.4 years) than patients $1,2,6,8,11$ and 12 (mean age 67.4 years). The first group has a greater functional capacity to compensate for a painful hip joint than the second group.

Patients $1,2,6,8,11$ and 12 have a rapid correction of |RDSLDS 3 months after arthroplasty. We explain it by the fact that pain is the responsible factor (before osteo-articular deformation and neuromuscular impairment) for the limp of osteo-arthritic patients before and after surgery. Three months after surgery, these patients had no more pain (pain is the principal indication for a hip arthroplasty) and almost no limp. Patient 9 is the only one still having hip pain at 6 months after surgery and has none at 9 months. This explains the slow progression of |RDS-LDS| after surgery.

Experiments performed on normal subjects have shown no significant difference between results obtained from accelerometry and those of pressure sensors attached under the foot. Additionally, accelerometers are less sensitive to artefacts present in pathological walking (e.g. foot shuffling, as shown in Fig. 4), as accelerations are measured on the leg and involve many advantages, such as high sensitivity, reproducibility, simple attachment, robustness and handling.

The error concerning the single and double support time depends on the mid-swing, heelstrike and toe-off detection time. This error is related to the time resolution of the system and parameters involved in peak detection (cutoff frequency of filters, threshold and intervals for peak searching). Errors reported in Table 2 show that the time resolution, which corresponds to the sampling period $(0.017 \mathrm{~s})$, is a major part of this error. By increasing the sampling rate, the resolution can be improved. However, a higher-frequency component of acceleration could be measured, and it is therefore necessary to adapt the cutoff frequency of filtering for mid-swing detection and the threshold to eliminate small peaks and enhance heelstrike and toe-off detection. A cutoff frequency of $3 \mathrm{~Hz}$ represents a good optimum in our tests. The intervals for heelstrikes and toe-off detection $\left(\left[p_{s} \pm 0.15 g c_{m}, p_{s} \pm 0.3 g c_{m}\right]\right)$ are fixed empirically in terms of mean gait cycle time; however, for very slow speeds, an increase in these intervals is necessary.

The proposed method for temporal gait parameter estimation using accelerometry reveals a promising monitoring tool for functional evaluation of gait improvement. The system used only two miniature low-cost accelerometers, with a light, portable (less than $300 \mathrm{~g}$ ) digital recorder, and involves no discomfort for the patient, who can carry it for a long period of time. Although the results introduced in this paper concern short-time measurement (a few minutes), the system allows long-term recording for up to $12 \mathrm{~h}$ during free living conditions. 
Table 2 Left stance (LS) and right stance (RS) obtained from accelerometers placed on each thigh (ACC) and FSR placed under each foot (FSR) as percentage of gait cycle, for 5 normal subjects

\begin{tabular}{|c|c|c|c|c|c|c|c|c|}
\hline & \multicolumn{2}{|c|}{ LS, s } & \multicolumn{2}{|c|}{ RS, s } & \multicolumn{2}{|c|}{ LS, \% } & \multicolumn{2}{|c|}{ RS, \% } \\
\hline & $\mathrm{ACC}$ & FSR & $\mathrm{ACC}$ & FSR & $\mathrm{ACC}$ & FSR & $\mathrm{ACC}$ & FSR \\
\hline 1 & $0.72 \pm 0.04$ & $0.73 \pm 0.03$ & $0.74 \pm 0.04$ & $0.73 \pm 0.03$ & $63 \pm 2$ & $63 \pm 2$ & $63 \pm 2$ & $62 \pm 2$ \\
\hline 2 & $0.61 \pm 0.02$ & $0.59 \pm 0.02$ & $0.64 \pm 0.02$ & $0.65 \pm 0.02$ & $60 \pm 2$ & $58 \pm 2$ & $60 \pm 2$ & $61 \pm 2$ \\
\hline 3 & $0.56 \pm 0.04$ & $0.57 \pm 0.03$ & $0.57 \pm 0.02$ & $0.58 \pm 0.02$ & $61 \pm 3$ & $62 \pm 2$ & $59 \pm 2$ & $61 \pm 2$ \\
\hline 4 & $0.56 \pm 0.02$ & $0.55 \pm 0.03$ & $0.59 \pm 0.01$ & $0.57 \pm 0.01$ & $62 \pm 3$ & $61 \pm 3$ & $63 \pm 2$ & $61 \pm 2$ \\
\hline 5 & $0.60 \pm 0.01$ & $0.63 \pm 0.01$ & $0.59 \pm 0.01$ & $0.61 \pm 0.01$ & $61 \pm 2$ & $63 \pm 2$ & $60 \pm 2$ & $62 \pm 2$ \\
\hline
\end{tabular}

By combining these temporal parameters with other gait features, such as movement co-ordination, distance parameters and pain level, a new functional score for gait improvement and treatment evaluation can be defined.

Acknowledgment-This research was supported by the Medical Engineering Research Program University Hospital, Federal Institute of Technology, University of Lausanne. The authors wish to acknowledge the contribution of M. J. Gramiger and M. P. Morel in the design of the portable measuring device.

\section{References}

ABU-Fara.J, Z. O., Harris, G. F., ABler, J. H., and WertsCh, J. J (1997): 'A Holter-type, microprocessor-based, rehabilitation instrument for acquisition and storage of plantar pressure data', $J$. Rehabil. Res. Dev., 34, pp 187-194

AMINIAN, K., ROBERT, P., JEQUier, E., and SCHUTZ, Y. (1995): 'Incline, speed, and distance assessment during unconstrained walking', Med. Sci. Sports Exerc., 27, pp. 226-234

AMinian, K., ROBERT, Ph., JÉQUIER, E., and SCHUTZ, Y. (1995): 'Estimation of speed and incline of walking using neural network', IEEE Trans. Inst. Meas., 44, pp. 743-746

AmInian, K., DE Andrès, E., ReZakhanlou, K., Fritsch, C., Depairon, M., Schutz, Y., LeYVRaz, P. F., and Robert, Ph. (1998): 'Motion analysis in clinical practice using ambulatory accelerometry, Captech 98, LNAI 1537, Springer-Verlag, pp. 1-11

ANDERSON, G. (1972): 'Hip assessment: a comparison of nine different methods, J. Bone Joint Surg.', 54B, pp. 621-625

BREIT, G. A., and WhALEN, R. T. (1997): 'Prediction of human gait parameters from temporal measures of foot-ground contact', Med. Sci. Sports Exerc., 29, pp. 540-547

CHAN, R. B., and CHILDRESS, D. S. (1991): 'Temporal factors of selfselected free-paces walking: relationships to an inverted pendulum method', Adv. Bioeng., 20, BED, ASME, pp. 525-530

EVANS, A. L., DUNCAN, G., and GILChRIST, W. (1991): 'Recording accelerations in body movements', Med. Biol. Eng. Comput., 29, pp. 102-104

FERrIGNO, G., and PEDOTTI, A. (1985): 'ELITE: A digital dedicated hardware system for movement analysis via real-time TV signal processing', IEEE Trans., BME-32, pp. 943-950

GIANNINI, S., CATANI, F., BENDETTI, M. G., and LEARDINI, A. (1994): 'Gait analysis: methodologies clinical applications', IOS Press, Amsterdam

GrauPE, D., GrosspietSCH, J. K., and Basseas, S. P. (1987): 'A single-microphone-based self-adaptive filter of noise from speech and its performance evaluation', J. Rehabil. Res. Dev., 24, pp. 119 126
HiroKaWA, S., and Matsumara, K. (1987): 'Gait analysis using a measuring walkway for temporal and distance factors', Med. Biol. Eng. Comput., 25, pp. 577-582

HOYT, R. W., KNAPIK, J. J., LANZA, J. F., JONES, B. H., and STAAB, J. S. (1994): 'Ambulatory foot contact monitor to estimate metabolic cost of human locomotion', J. Appl. Physiol., 76, pp. $1818-1822$

LIANG, M. H., FOSSET, A., and. LARSON, M. (1990): 'Comparisons of five health status instruments for orthopedic evaluation', Med. Care, 28, pp. 632-642

Lieberman, J. R., Dorey, F., Shekelle, P., Schumaker, L., ThOMAS, B. J., Kilgus, D. J., and. Finerman, G. A. (1996): 'Differences between patients' and physicians' evaluations of outcome after total hip arthroplasty', J. Bone Joint Surg., 78A, pp. $835-838$

MACEllari, V., and GiacomozzI, C. (1996): 'Multistep pressure platform as a stand-alone system for gait assessment', Med. Biol. Eng. Comput., 34, pp. 299-304

MAKI, B. E. (1997): 'Gait changes in older adults: predictors of falls or indicators of fear', J. Am. Geriatr. Soc., 453, pp. $313-320$

Rose, J., and GAMBle, J. (1994): 'Human walking' (Williams \& Wilkins, Baltimore), p. 29, p. 143

Rosenrot P. (1982): 'Footswitch stride analyser', Bull. Prosthet. Res., 18, p. 284

WALl, J. C., ASHBURN, A., and Klenerman, L. (1981): 'Gait analysis in the assessment of functional performance before and after total hip replacement', J. Biomed. Eng., 3, pp. 121-127

ZHU, H. S., Wertsch, J. J., HARRIS, G. F., LofTSGAarden, J. D., and PRICE, M. B. (1991): 'Foot pressure distribution during walking and shuffling', Arch. Phys. Rehabil., 72, pp. 390-397

\section{Author's biography}

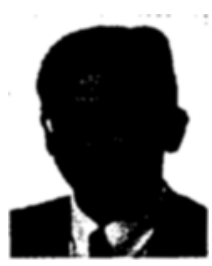

KAMIAR AMINIAN was born in Tehran, Iran, in 1958. He received his Electrical Engineering degree in 1982 and his $\mathrm{PhD}$ in the field of Biomedical Engineering in 1989 from the Swiss Federal Institute of Technology, Lausanne, Switzerland. Since 1982, he has worked in the Metrology Laboratory of the Swiss Federal Institute of Technology, Lausanne. From 1994 to 1996 he was Assistant Professor at the Sharif University of Technology, Tehran. In July 1996, he rejoined the Metrology Laboratory of Swiss Federal Institute of Technology, where he is head of the medical instrumentation group. His research interests include transducers, human body movement analysis, ambulatory systems for the measurement of physiological information and biomedical signal processing. 